\title{
MISCELANEA
}

COMUNICACIÓN PÓSTER VII CONGRESO NACIONAL DE LA SOCIEDAD CIENTÍFICA ESPAÑOLA DE ENFERMERÍA - SCELE. Mayo de 2014. UNIVERSIDAD DE ALICANTE.

\section{PERSPECTIVA ENFERMERA SOBRE LA COMPLEJIDAD DE LA COORDINACIÓN SOCIOSANITARIA EN LA ATENCIÓN DE ENFERMOS CRÓNICOS}

\section{PERSPECTIVE NURSE ABOUT THE COMPLEXITY OF COORDINATING HEALTHCARE IN THE CARE OF CHRONIC PATIENTS}

Lapeña-Moñux $\mathbf{Y R}^{1}$, Folch-Ayora $\mathbf{A}^{2}$, GonzálezChordá VM$^{3}$, Mena-Tudela ${ }^{4}$, Maciá-Soler ML $^{5}$, Pedraz-Marcos $\mathbf{A}^{6}$.

1-4Universidad Jaume I; 5Universidad de Alicante; ${ }^{6}$ Universidad Autónoma de Madrid 


\section{Palabras clave:} research

Disease management, primary health care, continuity of patient care, qualitative

\section{OBJETIVO:}

Describir la experiencia de los profesionales de enfermería en relación a las dificultades encontradas y los futuros retos de la coordinación asistencial dentro del Servicio Madrileño de Salud (SERMAS) para garantizar una atención integral al enfermo crónico (EC).

\section{MÉTODO:}

Estudio observacional-descriptivo con metodología cualitativa, realizado en el ámbito de la atención primaria del SERMAS, en 2013.

Los datos se obtuvieron mediante observación participante, entrevistas en profundidad y grupo de discusión, con profesionales sanitarios seleccionados mediante un muestreo intencional y amplia experiencia en el cuidado y gestión de EC.

Para el análisis de los datos se realizó un análisis temático siguiendo las cuatro fases propuestas por Morse y Field (1995).

En todo el proceso se tuvieron en cuenta los criterios de calidad descritos por Calderón (2002): adecuación epistemológica y metodológica, relevancia, validez y reflexividad.

\section{RESULTADOS:}

Los resultados se agrupan en 2 temas: (1) Dificultades para una coordinación de calidad y (2) retos en la coordinación sociosanitaria.

Observamos que existe un escaso desarrollo del marco político competencial sobre las estrategias que posicionan a la enfermería como el profesional clave de la atención al EC y permiten su visibilización social, de iniciativas de mejora basadas en evidencias científicas, múltiples barreras profesionales, organizativas, estructurales, de diseño tecnológico, relación y gestión que obstaculizan el cambio en la continuidad de cuidados.

Existen retos futuros dirigidos a empoderar a los enfermeros reconociendo su liderazgo por parte de la Administración, promover el trabajo colaborativo y adoptar un paradigma de asociación con éxito en los equipos multidisciplinares dirigidos por enfermeros y centrados en los pacientes, mejorar las estrategias de coordinación interniveles asistenciales garantizando eficiencia y calidad, fomentar el empoderamiento de pacientes mediante la autogestión de los cuidados, así como crear sistemas de comunicación e información compatibles. 